\title{
REPRÉSENTATIONS GALOISIENNES PAIRES
}

\author{
par M.-F. VIGNÉRAS
}

En honneur de Robert Rankin à l'occasion de son soixante-dixième anniversaire.

On présente des exemples de représentations de $G a l(\bar{Q} / Q)$ de dimension 2 , de déterminant pair, qui sont de type diédral (I) ou de conducteur premier et de type quelconque (II), en imitant la construction de Tate (Serre [11]) de représentations de déterminant impair.

On remarquera qu'une des applications est la construction explicite de formes modulaires non holomorphes, correspondant à la valeur propre $\lambda=1 / 4$ du Laplacien. En effet, si l'image de la représentation dans $\operatorname{PGL}(2, \mathbb{C})$ n'est pas isomorphe au groupe alterné $A_{5}$, la fonction $L$ d'Artin de la représentation est une fonction entière et sa transformée de Mellin (en un sens convenable) est une forme modulaire de poids 1, si le déterminant est impair, et une forme modulaire non holomorphe avec $\lambda=1 / 4$, si le déterminant est pair.

Un théorème de Deligne-Serre affirme que l'on obtient ainsi une bijection entre un ensemble de formes modulaires de poids 1 , et un ensemble de représentations galoisiennes de déterminant pair, satisfaisant la conjecture d'Artin. Un résultat analogue pour les formes modulaires non holomorphes, avec $\lambda=1 / 4$, semble encore hors d'atteinte.

1. Representations diédrales paires. On les obtient ainsi (Serre [11], §7, p. 237): on part d'un corps quadratique $K / \mathbb{Q}$, correspondant à un caractère $\omega$ du groupe de Galois $G_{Q}$ de $\overline{\mathbb{Q}} / \mathbb{Q}$, et d'une représentation $\chi$ de dimension 1 de $G_{K}$. Si $\sigma$ engendre $\operatorname{Gal}(K / \mathbb{Q})$ soit

$$
\chi_{\sigma}(\gamma)=\chi\left(\sigma \gamma \sigma^{-1}\right), \quad \gamma \in G_{K} .
$$

Soient f le conducteur de $\chi$ et $d$ le discriminant de $K$. Soit $\rho=\operatorname{Ind}_{K / \mathbb{Q}}(\chi)$ et $\tilde{\rho}$ la représentation projective associée. Alors:

1) $\rho$ est diédrale (ou irréductible) si et seulement si $\chi \neq \chi_{\sigma}$

2) le conducteur $N$ de $\rho$ est $|d|$. $N_{K / \mathbb{Q}}(\mathbf{f})$

3) $\rho$ est paire, $\rho$ est dite paire si son determinant est pair, si et seulement si $K$ est réel, et $\chi$ prend la même valeur $\varepsilon= \pm 1$ sur les deux Frobenius à l'infini. Si $c \in G_{\mathbb{Q}}$ est la conjugaison complexe, on a $\rho(c)=\varepsilon$ id.

4) $\operatorname{dét}(\rho)=\omega \chi_{\mathbb{Q}}$, où en termes d'idèles, $\chi_{\mathbb{Q}}$ est la restriction de $\chi$ aux classes d'idèles de $\mathbb{Q}$.

5) Si $\rho$ est diédrale, $\tilde{\rho}\left(G_{Q}\right)=D_{n}$, où $n$ est l'ordre de $\chi^{-1} \chi_{\sigma}$, et $D_{n}$ est le groupe diédral d'ordre $2 n$.

1.1. Représentations diédrales paires de conducteur premier $p, p<2000$. On déduit de ci-dessus:

1) $p \equiv 1 \bmod 4$

2) le nombre de classes $h$ de $K=\mathbb{Q}(\sqrt{p})$ est impair, différent de 1

Glasgow Math. J. 27 (1985) 223-237. 
3) $\varepsilon=1$ (par la théorie du genre)

4) dét $(\rho)$ comme caractère de Dirichlet est le symbole de Legendre $n \rightarrow\left(\frac{n}{p}\right)$

5) $\chi$ est un caractère non ramifié de $K, \chi^{2} \neq 1$ (la norme d'un idéal de $K$ étant principale, $\chi x^{\sigma}=1$ )

6) il y a $(h-1) / 2$ représentations diédrales irréductibles paires non équivalentes de conducteur $p$.

On les obtient en utilisant les tables numériques de Borevich-Chafarevich ([2]):

Il y a 143 nombres premiers $p, \quad p \equiv 1 \bmod 4, \quad p<2000$.

Le nombre de classes $h$ est égal à 3 pour les 8 nombres

$$
229,257,733,761,1229,1373,1489,1901 .
$$

Le nombre de classes $h$ est égal à 5 pour les 3 nombres

$$
401,1093,1429 .
$$

Le nombre de classes $h$ est égal à 7 pour les 3 nombres

$$
577,1009,1601 .
$$

On a $h=9$ pour $p=1129$, et $h=11$ pour $p=1297$.

$$
\text { Pour les } 127 \text { premiers restants, on a } h=1 \text {. }
$$

Donc, le nombre $n$ de représentations diédrales non isomorphes de conducteur premier $p<2000$ est égal à:

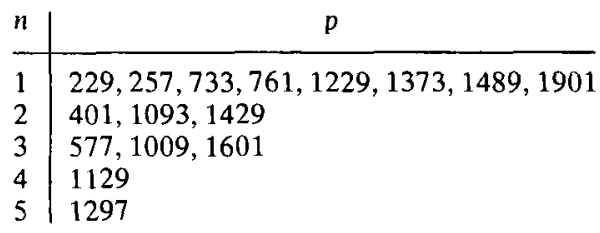

Il est égal à 0 pour les autres valeurs de $p, p<2000$.

A conjugaison près, $\mathrm{GL}(2, \mathbb{C})$ contient un seul sous-groupe $H_{n}$ isomorphe au groupe diédral $D_{n}$ : si $\xi$ est une racine primitive d'ordre $n$ de l'unité,

$$
\begin{aligned}
& H_{n}=\left\{\left(\begin{array}{ll}
\xi^{a} & \\
& \xi^{-a}
\end{array}\right),\left(\begin{array}{ll}
\xi^{-a} & \xi^{a}
\end{array}\right), 0 \leqslant a<n\right\}, \text { si } n \neq 2 . \\
& H_{2}=\left\{\left(\begin{array}{ll}
\mp 1 & \\
& \mp 1
\end{array}\right)\right\} .
\end{aligned}
$$

Si $n$ est impair, $\tilde{H}_{n} \simeq H_{n} \simeq D_{n}$, et si $n$ est pair, $\tilde{H}_{n} \simeq H_{n} /\{\mp 1\} \simeq D_{n / 2}$. A conjugaison près, il y a $\varphi(n)$ plongements de $D_{n}=\langle x, y\rangle, x^{n}=1, y^{2}=1, x y=y x^{-1}$ dans $\operatorname{GL}(2, \mathbb{C})$ si $n$ est impair.

Les 32 représentations diédrales paires de conducteur $p<2000$ premier s'obtiennent 
aussi de le façon suivante: soit $E$ l'extension abélienne maximale non ramifiée de $K$; elle est galoisienne sur $\mathbb{Q}$, de groupe de Galois $G_{E / \mathbb{Q}}$ isomorphe à $D_{h}$. Si $h \neq 1$, les représentations $\rho$ de conducteur $p$ s'obtiennent en composant la surjection canonique de $G_{Q}$ sur $G_{E / Q}$ avec les $(h-1) / 2$ plongements de $D_{h}$ dans $G L(2, \mathbb{C})$. Quand $h=3$, E est la clôture galoisienne d'un corps cubique non abélien totalement réel. Les tables numériques de Delone-Faddeev ([6]) donnent l'equation correspondante pour $p \leqslant 1229$. Les equations pour $p=1373,1489,1901$ m'ont été communiquées par J. Martinet.

\begin{tabular}{|c|c|}
\hline conducteur & équation \\
\hline 229 & $-4 x-1$ \\
\hline 257 & $x^{3}-x^{2}-4 x+3$ \\
\hline 733 & $x^{3}-x^{2}-7 x+8$ \\
\hline 761 & $x^{3}-x^{2}-6 x-1$ \\
\hline 1229 & $x^{3}-x^{2}-7 x-3$ \\
\hline 1373 & $-8 x+5$ \\
\hline 1489 & $x^{3}-8 x^{2}+9 x-1$ \\
\hline 1901 & $x^{3}+x^{2}-9 x+4$ \\
\hline
\end{tabular}

1.2. Représentations diédrales paires de conducteur $N \leqslant 200$. La représentation $\rho$ détermine le couple $(d, f)$ où $f=N_{K / \mathcal{O}}(\mathbf{f})$. On voit comme en 1 :

1) Il y a $\left(h-h_{2}\right) / 2$ représentations diédrales paires non isomorphes avec $N=d, f=1$, $\xi=1$, où $h_{2}$ est le nombre de classes d'idéaux de $K$ annulées par 2.

2) Il y a $\left(h^{+}-h_{2}^{+}\right) / 2-\left(h-h_{2}\right) / 2$ représentations diédrales paires non isomorphes avec $N=d, f=1, \varepsilon=-1$, où $h^{+}$est le nombre de classes d'idéaux au sens restreint de $K$ et $h_{2}^{+}$le nombre de ces classes qui sont annulées par 2.

Il y a 60 discriminants $d \leqslant 200$ de corps quadratiques réels. Les tables ([2]) montrent que

$$
\begin{aligned}
h & =\left\{\begin{array}{llll}
1 & \text { pour } & 46 & \text { discriminants } \\
2 & \text { pour } & 13 & \text { discriminants } \\
4 & \text { pour } & d=145
\end{array}\right. \\
h^{+} & =\left\{\begin{array}{llll}
1 & \text { pour } & 22 & \text { discriminants } \\
2 & \text { pour } & 29 & \text { discriminants } \\
4 & \text { pour } & d=60,105,120,136,140,145,156,165,168
\end{array}\right.
\end{aligned}
$$

La théorie du genre ([2]) montre $h_{2}^{+}=2^{t-1}$ où $t$ est le nombre de diviseurs premiers distincts de $d$. On en déduit que pour les nombres

$$
136,145
$$

le groupe des classes au sens restreint est isomorphe à $\mathbb{Z} / 4 \mathbb{Z}$.

Le corps de classes associé est une extension galoisienne non abélienne $E_{d} / \mathbb{Q}$ de degré 8 ( $\left.\operatorname{car} \chi \neq \chi^{\sigma}\right)$. Elle contient un corps biquadratique totalement réel $L_{d}$ (correspondant à la factorisation de $d$ en produit de deux discriminants $L_{136}=\mathbb{Q}(\sqrt{2}, \sqrt{17}), L_{145}=$ $\mathbb{Q}(\sqrt{5}, \sqrt{29})$ ). 
Il y a deux groupes non abéliens d'ordre 8:

- le groupe diédral $D_{4}$ ayant 3 sous-groupes distingués d'ordre 4, 5 sous-groupes d'ordre 2 (dont 2 distingués), 1 sous-groupe d'ordre 1 ,

- le groupe quaternionien $H_{8}=\langle i, j\rangle, i^{4}=1, i j=j i^{3}, i^{2}=j^{2}$, ayant 3 sous-groupes distingués d'ordre 4,1 sous-groupe distingué d'ordre 2 , sous-groupe d'ordre 1 . Il se plonge par un isomorphisme unique à conjugaison près dans $\mathrm{GL}(2, \mathbb{C})$ via les matrices de Pauli:

$$
i \rightarrow I=\left(\begin{array}{cc}
i & \\
& -i
\end{array}\right), \quad j \rightarrow J=\left(\begin{array}{rr} 
& -1 \\
1 &
\end{array}\right) .
$$

Son image dans $\operatorname{PGL}(2, \mathbb{C})$ est le groupe diédral $D_{2}$.

On en déduit que $\operatorname{Gal}\left(E_{d} / \mathbb{Q}\right)$ est isomorphe à $H_{8}$. Nous avons obtenu:

Il existe deux représentations diédrales paires non isomorphes avec $N=d \leqslant 200, f=1$. Pour l'une $N=136, \varepsilon=-1$, pour l'autre $N=145, \varepsilon=1$. On les obtient en composant la surjection canonique de $G_{\mathbb{Q}}$ sur $\mathrm{Gal}\left(E_{d} / \mathbb{Q}\right)$ avec le plongement de $H_{8}$ dans $\mathrm{GL}(2, \mathbb{C})$.

Si $f \neq 1$, on se propose de montrer:

Il existe une unique représentation diédrale paire, à isomorphisme près, avec $N \leqslant 200$, $f \neq 1$. Si $E / \mathbb{Q}$ est l'extension galoisienne de groupe de Galois $S_{3}$ engendrée par les racines du polynôme $x^{3}-x^{2}-3 x+1$, la représentation s'obtient en composant la surjection cononique de $G_{\mathbb{Q}}$ sur $\mathrm{Gal}(E / \mathbb{Q})$ avec le plongement unique à conjugaison près de $S_{3}$ dans $\mathrm{GL}(2, \mathbb{C})$.

Preuve. On fait les observations suivantes

1) $\chi$ n'est pas d'ordre 2, si $\rho$ est diédrale,

2) $\chi$ vu comme caractère d'idéaux, $\chi$ est trivial sur le groupe des idéaux principaux engendrés par un élément de $K_{\mathbf{f}}^{+}=\left\{a \in K^{*}, a \equiv 1 \bmod \mathbf{f}, N_{K / \mathbb{Q}}(a)>0\right\}$.

3) l'ordre $h_{\mathrm{f}}^{+} \mathrm{du}$ groupe des classes $C_{\mathrm{f}}^{+}$associé est donné par la formule:

$$
H_{\mathbf{f}}^{+}=h_{\mathbf{f}}^{2} /\left[U_{\mathbf{f}}: U_{\mathbf{f}}^{+}\right], \quad h_{\mathbf{f}}=h \varphi(\mathbf{f}) /\left[U: U_{\mathbf{f}}\right]
$$

où $U$ est le groupe des unités de $K, U_{\mathrm{f}}^{+}$le sous-groupe des unités de $K$ appartenant à $K_{\mathrm{f}}^{+}$, et $U_{\mathbf{f}}$ celles congrues à 1 modulo f. On rappelle que

$$
\varphi(\mathbf{f})=\prod N_{K / \mathbb{Q}}(P)^{m(P)-1}\left(N_{K / \mathbb{Q}}(P)-1\right) \quad \text { si } \quad \mathbf{f}=\prod P^{m(P)}
$$

4) soit $X$ un caractère de $C_{\mathrm{f}}^{+}$, identifié au groupe de Galois du corps de classes $K_{\mathrm{f}}^{+} / K$ associé; si $h_{\mathrm{f}}^{+}=h_{\mathrm{f}}^{+}$, alors $X$ prend la valeur +1 sur les deux Frobenius à l'infini; si $h_{f^{+}}^{+}=h_{\mathrm{f}}^{+}$ avec $\mathbf{f}^{\prime} \mid \mathbf{f}$, alors le conducteur de $X$ divise $\mathbf{f}^{\prime}$.

5) si $\mathbf{f}=\mathbf{f}^{\sigma}, K_{f}^{+} / \mathbb{Q}$ est galoisien, et il existe $X$ tel que $x \neq X^{\sigma}$, si et seulement si $K_{f}^{+} / \mathbb{Q}$ n'est pas abélien.

6) si $f=f^{\sigma}$, et si $F \geqslant 1$ engendre $\mathbf{f} \cap \mathbb{Z}$, alors $K_{\mathbf{f}}^{+} \supset \mathbb{Q}(\sqrt[F]{1})$ (si $A=(a) a \in K_{\mathbf{f}}^{+}$, alors $N_{\mathrm{K} / \mathbb{Q}}(A)=a a^{\sigma}, a, a^{\sigma} \in 1+f$, et $\left.a a^{\sigma} \in(1+f) \cap \mathbb{Z}=1+F\right)$.

On dresse alors la liste des 84 valeurs de $(d, f)$, avec $N=d f \leqslant 200$. On [calcule $h_{\mathbf{f}}$ et $h_{\mathbf{f}}^{+}$ pour les idéaux $\mathbf{f}$ de norme $f$. 
REPRÉSENTATIONS GALOISIENNES PAIRES

\begin{tabular}{|c|c|c|c|c|c|c|c|}
\hline$d$ & $f$ & $h_{\mathrm{f}}$ & $h_{\mathrm{l}}^{+}$ & $d$ & $f$ & $h_{\mathbf{f}}$ & $h_{\mathrm{f}}^{+}$ \\
\hline \multirow[t]{10}{*}{5} & 5 & 1 & 2 & & 2.3 & 1 & 2 \\
\hline & $5^{2}$ & 1 & 2 & & $5 *$ & 1 & 2 \\
\hline & $2^{2}$ & 1 & 1 & & & & \\
\hline & $\begin{array}{l}2^{4} \\
2^{2}\end{array}$ & 1 & 2 & 28 & 2 & 1 & 2 \\
\hline & $3^{2}$ & 1 & 2 & & $2^{2}$ & 1 & 2 \\
\hline & $2^{2} 3^{2}$ & 1 & 2 & & 7 & 3 & 6 \\
\hline & $2^{2} 5$ & 1 & 2 & & $3 *$ & 1 & 2 \\
\hline & $11 *$ & 1 & 1 & 0 & $2^{2}$ & 1 & 1 \\
\hline & $29_{*}$ & $\frac{1}{2}$ & $\begin{array}{l}1 \\
2\end{array}$ & 29 & 5 & 2 & 1 \\
\hline & $31_{*}$ & 1 & 1 & & & & \\
\hline \multirow{9}{*}{8} & & 1 & & 33 & $2 *$ & 1 & 2 \\
\hline & $2^{2}$ & $\begin{array}{l}1 \\
1\end{array}$ & $\begin{array}{l}1 \\
2\end{array}$ & & $2^{2}$ & 1 & 2 \\
\hline & $2^{3}$ & 1 & 2 & & $\begin{array}{l}* \\
3\end{array}$ & 1 & 2 \\
\hline & $2^{4}$ & 1 & 2 & & $2.3_{*}$ & 1 & 2 \\
\hline & $3^{2}$ & 1 & 2 & & & & \\
\hline & $5^{2}$ & 2 & 4 & 37 & $2^{2}$ & 3 & 3 \\
\hline & $2.3^{2}$ & 1 & 2 & & $3 *$ & 2 & 2 \\
\hline & $\begin{array}{l}7_{*} \\
17\end{array}$ & $\begin{array}{l}1 \\
1\end{array}$ & $\begin{array}{l}1 \\
2\end{array}$ & 30 & 2 & 2 & 2 \\
\hline & $23 *$ & 1 & 1 & & $2^{2}$ & 2 & 4 \\
\hline \multirow[t]{9}{*}{12} & 2 & 1 & 2 & & $3 *$ & 1 & $\begin{array}{l}4 \\
1\end{array}$ \\
\hline & $2^{2}$ & 1 & 2 & & & & \\
\hline & $2^{3}$ & 1 & 2 & 41 & 2 & 1 & 1 \\
\hline & $2^{4}$ & 2 & 4 & & $2^{2}$ & 1 & 1 \\
\hline & 3 & 1 & 2 & & $*$ & 1 & 1 \\
\hline & $3^{2}$ & 1 & 2 & & & & \\
\hline & $2^{2} \cdot 3$ & $\begin{array}{l}1 \\
1\end{array}$ & 2 & 44 & ${ }_{2}^{2}$ & 1 & 2 \\
\hline & $11_{*}$ & 1 & 2 & & & & \\
\hline & $13_{*}$ & 1 & 2 & 57 & 3 & 1 & 2 \\
\hline \multirow{4}{*}{13} & & & & & $2 *$ & 1 & 2 \\
\hline & 3 & 1 & 1 & 60 & 2 & 2 & 4 \\
\hline & $3 *$ & 1 & 1 & & 3 & 2 & 4 \\
\hline & & 1 & 2 & & & & \\
\hline \multirow{3}{*}{17} & $2 *$ & 1 & 1 & 61 & $3 *$ & 1 & 1 \\
\hline & $2^{2}$ & 1 & 1 & 65 & $2 *$ & 2 & 2 \\
\hline & $\begin{array}{l}* \\
3^{2}\end{array}$ & $\begin{array}{l}2 \\
1\end{array}$ & $\begin{array}{l}2 \\
2\end{array}$ & 73 & $2 *$ & 1 & 1 \\
\hline \multirow{5}{*}{21} & $2^{2}$ & 1 & 2 & 76 & 2 & 1 & 2 \\
\hline & 3 & 1 & 2 & & & & \\
\hline & $3^{2}$ & 1 & 2 & 88 & 2 & 1 & 2 \\
\hline & 7 & 3 & 6 & & & & \\
\hline & $5 *$ & 1 & 2 & 89 & $2 *$ & 1 & 1 \\
\hline \multirow[t]{3}{*}{24} & $\begin{array}{l}2 \\
2^{2}\end{array}$ & $\begin{array}{l}1 \\
2\end{array}$ & $\begin{array}{l}2 \\
4\end{array}$ & 92 & 2 & 1 & 2 \\
\hline & $2^{3}$ & 2 & $\begin{array}{l}4 \\
4\end{array}$ & 97 & $2 *$ & 1 & 1 \\
\hline & 3 & 1 & 2 & & & & \\
\hline
\end{tabular}


Dans le tableau ci-dessus, $*$ signifie que $f \neq f^{\sigma}$. Utilisant 1 ) on ne retient que les 11 valeurs de $(d, f)$ où $h>2$. On calcule $K_{f}^{+}$dans chaque cas.

\begin{tabular}{rllll}
\hline$d$ & $f$ & $h_{f}$ & $h_{f}^{+}$ & $K_{f}^{+}$ \\
\hline 8 & $5^{2}$ & 2 & 4 & $\mathbb{Q}(\sqrt{2}, \sqrt[51]{1})$ \\
12 & $2^{4}$ & 2 & 4 & $\mathbb{Q}(\sqrt{3}, \sqrt{-1})$ \\
21 & 7 & 3 & 6 & $\mathbb{Q}(\sqrt{21}, \sqrt[7]{1})$ \\
24 & $2^{2}$ & 2 & 4 & $\mathbb{Q}(\sqrt{6}, \sqrt{-1}, \sqrt{-2})$ \\
& $2^{3}$ & 2 & 4 & $\mathbb{Q}(\sqrt{6}, \sqrt{-1}, \sqrt{-2})$ \\
28 & 7 & 3 & 6 & $\mathbb{Q}(\sqrt{-1}, \sqrt[7]{1})$ \\
37 & $2^{2}$ & 3 & 3 & corps engendré par les racines du polynôme \\
& & & & $x^{3}-x^{2}-3 x+1$, de groupe de Galois sur $\mathbb{Q}$ égal à $D_{3}$ \\
40 & $2^{2}$ & 2 & 4 & $\mathbb{Q}(\sqrt{10}, \sqrt{2}, \sqrt{-1})$ \\
& 5 & 2 & 4 & $\mathbb{Q}(\sqrt{10}, \sqrt[5]{1})$ \\
60 & 2 & 2 & 4 & $K^{+}=\mathbb{Q}(\sqrt{15}, \sqrt{5}, \sqrt{-3})$ \\
& 3 & 2 & 4 & $K^{+}=\mathbb{Q}(\sqrt{15}, \sqrt{5}, \sqrt{-3})$ \\
\hline
\end{tabular}

Tous les corps $K_{f}^{+}$sont abéliens sauf celui correspondant à $N=148$.

1.3. Représentations diédrales paires associées à un corps cubique non abélien totalement réel. Un corps cubique non abélien totalement réel $L / Q$ produit une représentation diédrale $\rho$ avec les propriétés suivantes:

1) si $E$ est la clôture galoisienne de $L$, alors $G a l(E / \mathbb{Q})$ est isomorphe à $D_{3}$ et $\rho$ est le composé de la surjection naturelle de $G_{\mathbb{Q}}$ sur $G a l(E / \mathbb{Q})$ et du plongement, unique à conjugaison près, de $D_{3}$ dans $G L(2, \mathbb{C})$.

2) soit $D$ le discriminant de $L / \mathbb{Q}$, et $K=\mathbb{Q}(\sqrt{D})$ de discriminant $d$. Alors $E=K L$, et $\rho=\operatorname{Ind}_{G_{K}}^{G_{a}}(\chi)$, où $\chi$ est le composé de la surjection canonique de $G_{K} \operatorname{sur} G a l(E / K)$ et d'un caractère non trivial de $\mathrm{Gal}(E / K)$.

3) $\varepsilon=1$. $E / K$.

4) $N=d f$, où l'idéal $(f)_{K}$ engendré par $f$ dans $K$ est égal au discriminant $\Delta_{E / K}$ de

5) Soit $N=\prod p^{n(p)}$ et $D=\prod p^{m(p)}$; on a $m(p)=n(p)$ si $p \neq 2$ ou 3 , $m(3)=n(3)$ si et seulement si $m(3) \leqslant 3$ (par [6] p. $217, m(3) \leqslant 5)$, $m(2)=n(2)$ si et seulement si 2 n'est pas totalement ramifié dans $L$ quand on a simultanément $m(2)=2$ et $2 \mid d$ (par [6], $m(2)=2$ ou 3 ).

6) En général $N$ est donné par la formule:

$$
N=d \prod p^{m(p)} \prod q^{m(q)-1}
$$

où $p$ parcourt les premiers totalement ramifiés dans $L$, non ramifiés dans $K$ et $q$ ceux totalement ramifiés dans $L$ et dans $K$.

Preuve. Vérifions la formule, le reste se déduit de ce qui précède. On a les relations 
de transitivité entre différentes et discriminants:

$$
\mathscr{D}_{E / \mathbb{Q}}=\mathscr{D}_{E / K} \mathscr{D}_{\mathrm{K} / \mathbb{Q}}=\mathscr{D}_{E / L} \mathscr{D}_{L / Q}, \quad \Delta_{E / Q}=\left(\Delta_{E / K}\right)^{2} d^{3} .
$$

Si $p$ premier ne se ramifie pas dans $L$, il ne divise pas $\Delta_{E / K}$.

$\mathrm{Si}(p)_{\mathrm{L}}=\gamma^{2} Q$, est la décomposition en idéaux premiers de l'idéal engendré par $p$ dans $L$, alors $p$ ne divise pas $\Delta_{E / K}$.

Supposons donc $(p)_{L}=\gamma^{3}$. Alors:

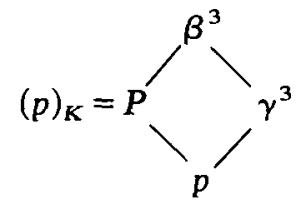

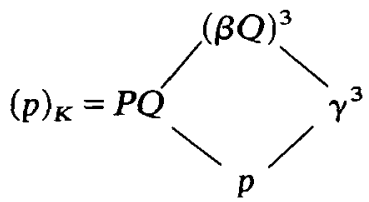<smiles>[Te]=P12[B][P+]1P2</smiles>

Si $p \neq 3$, la ramification de $E / K$ en $\beta \mid p$ est modérée et l'exposant de $\beta$ dans $\mathscr{D}_{E / K}$ est égal à 2 .

Si $p=3$, et si $m(3) \geqslant 3$, l'exposant de $\beta \mid 3$ dans $\mathscr{D}_{E / K}$ est egal à $m(p)$ si $p \nmid d$, et $2(m(p)-1)$ si $p \mid d$. Prenant la norme sur $K$, on obtient la formule.

Pour les 28 corps cubiques non abéliens totalement réels donnés par les tables ([6]), on a $N=D$.

\begin{tabular}{|c|c|c|c|c|}
\hline Discriminant & Equation & $d$ & $f$ & $N$ \\
\hline $148=2^{2} .37$ & $x^{3}-x^{2}-3 x+1$ & 37 & $2^{2}$ & 148 \\
\hline 229 & $-4 x$ & 229 & - & 229 \\
\hline 257 & $x^{3}-x^{2}-4 x+3$ & 257 & 1 & 257 \\
\hline $316=2^{2} .79$ & $x^{3}-x^{2}-4 x+2$ & $2^{2} .79$ & 1 & 316 \\
\hline $321=3.107$ & $x^{3}-x^{2}-4 x+1$ & 3.107 & 1 & 321 \\
\hline $404=2^{2} .101$ & $x^{3}-x^{2}-5 x-1$ & 101 & $2^{2}$ & 404 \\
\hline $469=7.67$ & $x^{3}-x^{2}-5 x+4$ & 7.67 & 1 & 469 \\
\hline $473=11.43$ & $-5 x-1$ & 11.43 & 1 & 473 \\
\hline $564=2^{2} \cdot 3 \cdot 47$ & $x^{3}-x^{2}-5 x+3$ & 3.47 & $2^{2}$ & 564 \\
\hline $568=2^{3} .71$ & $x^{3}-x^{2}-6 x-2$ & $2^{3} .71$ & 1 & 568 \\
\hline $621=3^{3} .23$ & $-6 x-3$ & 3.23 & $3^{2}$ & 621 \\
\hline $697=17.41$ & $-7 x$ & 17.41 & 1 & 697 \\
\hline 733 & $x^{3}-x^{2}-7 x+8$ & 733 & 1 & 733 \\
\hline $756=2^{2} \cdot 3^{3} \cdot 7$ & $-6 x-2$ & 3.7 & $2^{2} \cdot 3^{2}$ & 756 \\
\hline 761 & $x^{3}-x^{2}-6 x-1$ & 761 & 1 & 761 \\
\hline $785=5.157$ & $x^{3}-x^{2}-6 x+5$ & 5.157 & 1 & 785 \\
\hline $788=2^{2} \cdot 197$ & $x^{3}-x^{2}-7 x-3$ & 197 & $2^{2}$ & 488 \\
\hline $837=3^{3} \cdot 31$ & $-6 x-1$ & 3.31 & $3^{2}$ & 837 \\
\hline $892=2^{2} .223$ & $x^{3}-x^{2}-8 x+10$ & $2^{2} .223$ & 1 & 892 \\
\hline $940=2^{2} .5 .47$ & $-7 x-4$ & $2^{2} \cdot 5 \cdot 47$ & 1 & 940 \\
\hline $985=5.197$ & $x^{3}-x^{2}-6 x+1$ & 5.197 & 1 & 985 \\
\hline $993=3.331$ & $x^{3}-x^{2}-6 x+3$ & 3.331 & 1 & 993 \\
\hline $1016=2^{3} .127$ & $x^{3}-x^{2}-6 x+2$ & $2^{3} \cdot 127$ & 1 & 1016 \\
\hline $1076=2^{2} .269$ & $-8 x-6$ & 269 & $2^{2}$ & 1076 \\
\hline $1101=3.367$ & $x^{3}-x^{2}-9 x+12$ & 3.367 & 1 & 1101 \\
\hline 1129 & $-7 x-3$ & 1129 & 1 & 1129 \\
\hline 1229 & $x^{3}-x^{2}-7 x+6$ & 1229 & 1 & 1229 \\
\hline $1257=3.419$ & $x^{3}-x^{2}-8 x+9$ & 3.419 & 1 & 1257 \\
\hline
\end{tabular}


2. Représentations de conducteur premier paires. Soit $\rho: G_{\mathbb{Q}} \rightarrow \mathrm{GL}(V)$ une représentation de $G_{\Omega}$ dans un espace vectoriel complexe $V$ de dimension 2 , de conducteur un nombre premier $p$. Alors det $\rho$ est une représentation de $G_{\mathbb{Q}}$ de dimension 1 , non triviale, de conucteur premier p. En effet, la formule du conducteur (Martinet [10], p. 22)

$$
\sum_{i \geqslant 0} g_{i} / g_{0} \operatorname{codim} V^{G_{i}}=1
$$

où $g_{i}$ est l'ordre du $i$-ème groupe de ramification $G_{i}$ de $\operatorname{Gal}(\operatorname{Ker} \rho / \mathbb{Q})$ en un idéal $P \mid p$, donne $G_{i}=\{1\}$ pour $i \geqslant 1$, et codim, $V^{G_{0}}=1$. Si $I_{p} \subset G_{Q}$ est le groupe d'inertie en une place de $\overline{\mathbb{Q}}$ sur $p$, on a:

$$
\left.\rho\right|_{I_{v}}=1 \oplus \psi, \quad \text { dét }\left.\rho\right|_{I_{p}}=\psi
$$

où $\psi$ est une représentation de dimension 1 , non triviale de $I_{p}$. Si $q$ est un premier, $q \neq p$, alors $\rho$ (restreint à $I_{q}$ est trivial. On a $p \neq 2$.

Si $\rho$ est réductible, alors $\rho=1 \oplus \chi$ où $\chi$ est une représentation de dimension 1 de $G_{Q}$, de conducteur $p$. Le nombre de représentations réductibles de dimension 2 , de conducteur premier $p \neq 2$ est égal à $p-1$. Il y en $a(p-1) / 2$ avec dét $\rho$ pair.

Il existe $(h-1) / 2$ représentations irréductibles non isomorphes $\rho$ de dimension 2 de $G_{Q}$, de conducteur premier $p \neq 2$, telles que $\tilde{\rho}\left(G_{\mathbb{Q}}\right)$ soit un groupe diédral;

1) si $p \equiv 1 \bmod 4$, dét $\rho$ est pair, $h$ est le nombre de classes de $\mathbb{Q}(\sqrt{p})(I)$

2) si $p=3 \bmod 4$, dét $\rho$ est impair, $h$ est le nombre de classes de $\mathbb{Q}(\sqrt{-p})([11])$ Le caractère dét $\rho$ est le symbole de Legendre $n \rightarrow\left(\frac{n}{p}\right)$.

On suppose désormais $\rho$ irréductible, non diédrale, alors det $\rho\left(G_{\mathbb{Q}}\right)$ est un sous-groupe fini de $\operatorname{PGL}(2, \mathbb{C})$ isomorphe à $A_{4}, S_{4}$, ou $A_{5}$. On suppose aussi que dét $\rho$ est pair. Serre a traité le cas où dét $\rho$ est impair. Ses méthodes s'étenden bien. Les isomorphismes canoniques:

$$
\text { dét } \rho\left(G_{Q}\right)=\operatorname{dét~} \rho\left(I_{\mathrm{p}}\right)=\rho\left(I_{\mathrm{p}}\right)=\tilde{\rho}\left(I_{\mathrm{p}}\right)
$$

montre que dét $\rho\left(G_{\odot}\right)$ est un sous-groupe cyclique de $A_{4}, S_{4}$ ou $A_{5}$, non trivial, donc d'ordre $2,3,4$ ou 5 . Comme dét $\rho$ est pair, on peut le voir comme und caractère de $(\mathbb{Z} / p \mathbb{Z})^{\times}$trivial sur l'image de -1 .

1) si dét $\rho$ est d'ordere 2 , alors dét $\rho$ est le symbole de Legendre $n \rightarrow\left(\frac{n}{p}\right), p \equiv 1 \bmod 4$, et $\rho$ est de type $S_{4}$ ou $A_{5}$

2) si dét $\rho$ est d'ordre 3 , alors $p \equiv 1 \bmod 3$, et $\rho$ est de type $A_{4}$ ou $A_{5}$

3) si dét $\rho$ est $d$ 'ordre 4 , alors $p \equiv 1 \bmod 8$, et $\rho$ est de type $S_{4}$

4) si dét $\rho$ est d'ordre 5, alors $p \equiv 1 \bmod 5$, et $\rho$ est de type $A_{5}$

En effet, comme $A_{4}$ et $A_{5}$ n'ont pas d'éléments d'ordre 4, comme $A_{4}$ et $S_{4}$ n'ont pas d'éléments d'ordre 5 , on a 3 ) et 4 ). Si dét $\rho$ était d'ordre 3 , et $\rho$ de type $S_{4}$, l'application composée:

$$
I_{\mathrm{p}} \rightarrow S_{4} \rightarrow S_{4} / A_{4}
$$


serait triviale, et le noyau de la composition de $G_{\mathbb{Q}} \rightarrow S_{4} \rightarrow S_{4} / A_{4}$ correspondrait à un corps quadratique partout non ramifié, ce qui est impossible. On démontre de même ([11]) que dét $\rho$ d'ordre 2 , et $\rho$ de type $A_{4}$ est impossible (utiliser la surjection $\left.A_{4} \rightarrow \mathbb{Z} / 3 \mathbb{Z}\right)$. On déduit aussi:

5) si $p \equiv 23,47$ ou $59 \bmod 60$, il n'existe pas de représentations irréductible non diédrales de dimension 2 de $G_{Q}$ de conducteur $p$.

Inversement, soit $p$ un nombre premier, et $E / \mathbb{Q}$ une extension galoisienne. On considère les cas suivants:

$$
\begin{gathered}
\mathrm{Gal}(E / \mathbb{Q})=A_{4} \text { et } p \equiv 1 \bmod 3 \\
\mathrm{Gal}(E / \mathbb{Q})=S_{4} \text { et } p \equiv 5 \bmod 8 \\
\mathrm{Gal}(E / \mathbb{Q})=S_{4} \text { et } p \equiv 1 \bmod 8 \\
\mathrm{Gal}(E / \mathbb{Q})=A_{5} \quad \text { et } \quad a(p \equiv 1 \bmod 5), \quad b(p \equiv 1 \bmod 3), \quad c(p \equiv 1 \bmod 4) \quad\left(3_{\mathrm{a}, \mathrm{b}, \mathrm{c}}\right)
\end{gathered}
$$
où $\mathrm{a}, \mathrm{b}, \mathrm{c} \in\{0,1\}$ et $0(A)=$ non $A, 1(A)=A$.

Soit $\tilde{\rho}_{E}$ le composé de la surjection canonique de $G_{\mathbb{Q}}$ dans $G a l(E / \mathbb{Q})$ et d'un plongement de $\operatorname{Gal}(E / \mathbb{Q})$ dans $\operatorname{PGL}(2, \mathbb{C})$.

THÉOREME. $\tilde{\rho}_{\mathrm{E}}$ admet un relèvement $\rho \mathrm{E}: G_{\mathbb{Q}} \rightarrow \mathrm{GL}(2, \mathbb{C})$ de conducteur $p$, avec dét $\rho_{\mathrm{E}}$ pair si et seulement si:

(1) E est la clôture galoisienne d'un corps quartique totalement réel $E_{4}$ de discriminant $p^{2}$.

$\left(2_{\mathrm{a}}\right)$ E est la clôture galoisienne d'un corps quartique totalement réel $E_{4}$ de discriminant $p$.

$\left(2_{b}\right)$ E est la clôture galoisienne d'un corps quartique totalement réel $E_{4}$ de discriminant p ou $p^{3}$.

$\left(3_{\mathrm{a}, \mathrm{b}, \mathrm{c}}\right)$ E est la clôture galoisienne d'un corps quintique totalement réel $E_{5}$ de discriminant $\mathrm{a}\left(p^{4}\right)$ ou $\mathrm{b}\left(p^{2}\right.$ et la décomposition en idéaux premiers de l'idéal engendré par $p$ dans $E_{5}$ est du type $P^{3} Q$ ou $\left.P^{3} Q R\right)$ ou $\mathrm{c}\left(p^{2}\right.$ et la décomposition de l'idéal engendré par $p$ dans $E_{5}$ n'est pas du type précédent).

Preuve. La condition $E$ totalement réel est équivalente à dét $\rho$ pair, pour un (= pour tout) relèvement $\rho$ de $\tilde{\rho}_{\mathrm{E}}$. Les conditions dur le discriminant et sur la ramification de $p$ sont nécessaires d'après les conditions 1 ) à 4 ) vues précédement. En effet, si $G_{0}$ est groupe d'inertie de $p$ dans $\operatorname{Gal}(E / \mathbb{Q})$, elles sont équivalentes à:

(1) $G_{0}$ est cyclique d'ordre $3($ comme $p \equiv 1 \bmod 3, E / \mathbb{Q}$ est modérément ramifié en $p$, et $G_{0}$ est un groupe cyclique d'ordre 2, 3 ou 6 . Comme $G_{0} \subset A_{4}, 6$ est impossible; il n'est pas d'ordre 2, sinon l'extension cubique cyclique $L / Q$ contenue dans $E$ serait partout non ramifiée, ce qui est impossible).

$\left(2_{\mathrm{a}}\right) G_{0}$ est cyclique d'ordre $2(p \equiv 5 \bmod 8$ montre que $E / \mathbb{Q}$ est modérément ramifiée, et $G_{0}$ est un groupe cyclique d'ordre 2 ou 6 . Comme $G_{0} \subset S_{4}, 6$ n'est pas possible).

$\left(2_{\mathrm{b}}\right) G_{0}$ est cyclique d'order 2 ou 4 (si le discriminant est $p^{3}, G_{0}$ est un groupe cyclique d'ordre $4 d, d \mid 6$. Etant contenu dans $S_{4}$, il est d'ordre 4).

$\left(3_{\mathrm{a}, \mathrm{b}, \mathrm{c}}\right) G_{0}$ est cyclique d'ordre a(5) ou b(3) ou c(2) (si le discriminant est $p^{4}$, et $p \equiv 1 \bmod 5, G_{0}$ est un groupe cyclique d'ordre $5 d, d \mid 12$. Comme $G_{0} \subset A_{5}, G_{0}$ est 
d'ordre 5; si le discriminant est $p^{2}$, et $p \equiv 1 \bmod 4$ ou $\bmod 3$, alors $G_{0}$ est un groupe cyclique d'ordre $2 d, d \mid 6$ ou $3 d, d \mid 4$. Comme $G_{0} \subset A_{5}, G_{0}$ est d'ordre 2 ou 3 . Les deux cas sont possibles; pour les distinguer, il suffit de connaître la décomposition de l'idéal premier engendré par $p$ dans $E_{5}$ ).

D'après ([11], p. 248-250) ces conditions sont suffisantes. Si Card $\left(G_{0}\right) \geqslant 3$, comme Card $\left(G_{0}\right)$ divise $p-1$, on applique le lemme ([11[, p. 248); si $\operatorname{Card}\left(G_{0}\right)=2$, on raisonne comme dans ([11], p. 249-250).

Quand les conditions du théorème sont satisfaites, dans chaque cas, $\tilde{\rho}_{E}$ admet deux relèvements irréductibles non isomorphes, de conducteur $p$, de déterminant pair, si $\rho$ est l'un d'eux, l'autre est $\bar{\rho}=\rho \oplus \operatorname{dét}(\rho)^{-1}$.

Le nombre de classes de conjugaison de plongements de $A_{4}$, resp. $S_{4}, A_{5}$ dans $\operatorname{PGL}(2, \mathbb{C})$ est 1 , resp. 1,2 . Il existe donc:

$2 x$ représentations irréductibles de dimension 2 de $G_{Q}$ de type $A_{4}$

$2 y$ représentations irréductibles de dimension 2 de $G_{\mathbb{Q}}$ de type $S_{4}$

$4 z$ représentations irréductibees de dimension 2 de $G_{Q}$ de type $A_{s}$

non isomorphes, de conducteur $p$, de déterminant pair, où $x$, (resp. $y, z$ ), est le nombre de corps quartiques, (resp. quartiques, quintiques) satisfaisant les hypothèses du théorème, cas (1), (resp. cas $2_{\mathrm{a}}$ ou $2_{\mathrm{b}}$ selon p, cas $3_{\mathrm{a}, \mathrm{b}, \mathrm{c}}(\mathrm{a}, \mathrm{b}, \mathrm{c})$ étant déterminé par $p$ ).

EXEMPLES NUMERIOUeS. On note $\rho$ une représentation de dimension 2 de $G_{Q}$ de déterminant pair, de conducteur un nombre premier $p$.

Les tables de corps quintiques de Buhler ([1]) semblent indiquer que:

$$
\text { Pour } p<10000 \text {, il n'existe aucun } \rho \text { de type } A_{5} \text {. }
$$

Les tables de corps quartiques totalement réels $E_{4} / Q$, ne contenant pas de sous-corps quadratique (condition nécessaire et suffisante pour que la clôture galoisienne $E / Q$ de $E_{4}$ ait un groupe de Galois isomorphe à $A_{4}$ (si le discriminant de $E_{4} / \mathbb{Q}$ est un carré) ou $S_{4}$ (sinon)) de Godwin ([8] p. 484) montrent que

(1) pour $p<106$, il n'existe aucun $\rho$ de type $A_{4}$,

(2) pour $p<11348$, il existe $10 \rho$ non isomorphes de type $S_{4}$ avec dét $\rho$ égal au symbole de Legendre. Les 5 valeurs de p sont:

$$
\text { 2777, 7537, 8069, 10273, 10889, } 11197 .
$$

Si $p \equiv 1 \bmod 8$, et $E_{4} / \mathbb{Q}$ de discriminant $p^{3}$, alors $\mathbb{Q}(\sqrt{p}) \subset E$ a un nombre de classes divisible par 3. Pour $p<2000$, il y a trois valeurs possibles ([2])

$$
p=257,761,1489 .
$$

Je ne sais pas si elles fournissent des exemples.

$\left(2_{\mathrm{b}}\right)$ pour $p \equiv 1 \bmod 8, p<2000, p \neq 257,761,1489$, il n'existe pas de $\rho$ de type $S_{4}$.

Application: représentations paires de conducteur premier $p$, de déterminant égal au symbole de Legendre $n \rightarrow\left(\frac{n}{p}\right), p \equiv 1 \bmod 4$. 
Il y en a:

1) 1 réductible,

2) $(h-1) / 2$ diédrales où $h$ est le nombre de classes de $\mathbb{Q}(\sqrt{p})$,

3) $2 x$ de type $S_{4}$ où $x$ est le nombre de corps quartiques totalement réels ne contenant pas de sous-corps quadratiques, et de discriminant $p$,

4) $4 s$ de type $A_{5}$, où $s$ est le nombre de corps quintiques $E_{5} / \mathbb{Q}$

(a) de discriminant $p^{2}$

(b) tels que $E_{5} \mathbb{Q}(\sqrt{p}) / \mathbb{Q}$ ne soit pas galoisien (par exemple, si $5+h$ )

(c) tels que l'indice de ramification d'un idéal premier $P \mid p$ de $E_{5}$ soit inférieur ou égal à 2 .

Le groupe de Galois de la clôture galoisienne $E / \mathbb{Q}$ de $E_{5}$ est un sous-groupe de $A_{5}$, différent de $\mathbb{Z} / 5 \mathbb{Z}$, par (a) et de $D_{5}$ par (b), donc égal à $A_{5}$. Par (c), le groupe d'inertie de $\mathrm{Gal}(E / \mathbb{Q})$ en $p$ est d'ordre 2.

Si $\rho$ est réductible, ou diédrale, la conjugaison complexe opère trivialement; je ne sais pas si elle opère trivialement dans les autres cas.

\section{Fonctions $L$}

3.1. Représentations galoisiennes de dimension 2. Soit $\rho$ une représentation irréductible, linéaire, complexe, de dimension 2 de $G_{\mathbb{Q}}$, de déterminant pair, telle que $\rho(c)=(-1)^{a}$ id., où $c \in G_{\mathbb{Q}}$ est une conjugaison complexe, et $a=0$ ou 1 .

Soit $N(\rho)$ le conducteur d'Artin et $L(s, \rho)$ la fonction d'Artin de la représentation $\rho$ (on réfère à $[\mathbf{1 0}]$ p. 7 à 18 pour leurs définitions). On pose

$$
\Lambda(s, \rho)=N(\rho)^{s / 2} \pi^{-(s+a)} \Gamma\left(\frac{s+a}{2}\right)^{2} L(s, \rho) .
$$

Alors, on sait que $\Lambda$ s'étend en une fonction méromorphe de $s$ sur tout le plan complexe et vérifie l'équation fonctionnelle:

$$
\Lambda(1-s, \rho)=W(\rho) \Lambda(s, \bar{\rho})
$$

où $\bar{\rho}$ est la contragrédiente de $\rho$, et $W(\rho)$ une constante de module 1 . La conjecture d'Artin dit que $\Lambda$ est une fonction holomorphe de $S$. Il est utile d'introduire la condition (A):

(A) Il exite un entier $M$ tel que pour toute représentation linéaire continue $\chi$ de dimension 1 de $G_{Q}$, de conducteur premier à $M$, la fonction $\Lambda(s, \rho \otimes \chi)$ est holomorphe en $s$.

La représentation vérifie la condition (A) si l'image de $\rho$ dans $\operatorname{PGL}(2, \mathbb{Q})$ n'est pas isomorphe à $A_{5}$ (c'est bien connu si $\rho$ est de type diédral, c'est un célèbre résultat de Langlands si $\rho$ est de type $A_{4}$, le résultat pour $\rho$ de type $S_{4}$ se déduit de celui pour $A_{4}$ comme l'a démontré Tunnell ([12]).

3.2. Représentations automorphes de GL(2). Soit. $A_{\mathbb{Q}}$ l'anneau des adèles de $\mathbb{Q}$; soit $\pi$ une représentation automorphe cuspidale de $\mathrm{GL}\left(2, A_{\mathscr{Q}}\right)$, autrement dit un composant irréductible de l'action naturelle de $\mathrm{GL}\left(2, A_{\mathbb{Q}}\right)$ dans l'ensemble des fonctions sur 
$\mathrm{GL}(2, \mathbb{Q}) \backslash \mathrm{GL}\left(2, A_{\mathbb{Q}}\right)$ satisfaisant des conditions raisonnables (pour les définitions, on refère à [3]). On écrit $\pi$ comme un produit tensoriel restreint

$$
\pi=\otimes \pi_{\mathrm{v}}
$$

de représentations irréductibles admissibles unitaires $\pi_{v}$ de $G L\left(2, \mathbb{Q}_{v}\right)$, où $v$ parcourt les places de $\mathbb{Q}$, et $\mathbb{Q}_{v}$ est le complété de $\mathbb{Q}$ en $v$. On suppose que pour la place archimédienne $v=\infty$, la représentation $\pi_{\infty}$ est la représentation, dite de la série principale, définie par l'une des propriétés équivalentes suivantes ([5]):

1) $\pi_{\infty}$ est la représentation obtenue par le foncteur d'induction unitaire à partir de la représentation continue, de dimension 1 , du groupe triangulaire supérieur:

$$
\left(\operatorname{sign}^{a} \otimes \operatorname{sign}^{a}\right)\left(\begin{array}{ll}
x & y \\
0 & z
\end{array}\right)=(\operatorname{sign} x)^{a}(\operatorname{sign} z)^{a}
$$

2) $\pi_{\infty}$ est l'unique représentation irréductible admissible de $\operatorname{GL}(2, \mathbb{R})$ telle que

- la restriction de $\pi_{\infty}$ au centre de $G L(2, \mathbb{R})$ est triviale,

- il existe dans l'espace de $\pi_{\infty}$ un vecteur $v$ invariant par $\operatorname{SO}(2, \mathbb{R})$ et tel que

$$
\pi_{\infty}\left(\begin{array}{cc}
1 & \\
& -1
\end{array}\right) v=(-1)^{a} v
$$

- soit $C$ l'opérateur de Casimir standard dans l'algèbre enveloppante de GL(2, R) alors $\pi_{\infty}(C)=-1 / 4$.

La fonction $L\left(s, \pi_{\infty}\right)$ associée à $\pi_{\infty}$ est égale à:

$$
L\left(s, \pi_{\infty}\right)=\pi^{-(s+a)}((s+a) / 2)^{2} .
$$

On définit le conducteur $N(\pi)$, la fonction $L(s, \pi)$ et le facteur $\varepsilon(s, \pi)$ de la représentation $\pi$; on a

$$
\varepsilon(s, \pi)=N(\pi)^{(-s+1 / 2)} W(\pi),
$$

où $W(\pi)$ est une constante de module 1 . Jacquet et Langlands ont montré que la fonction $L$ se prolonge en une fonction holomorphe de $s$ sur le plan complexes et vérifie une équation fonctionnelle:

$$
L(s, \pi)=\varepsilon(s, \pi) L(1-s, \tilde{\pi}),
$$

où $\tilde{\pi}$ est la représentation contragrédiente de $\pi$. Si $\omega(\pi)$ est le caractère central associé à $\pi$, alors $\tilde{\pi}=\pi \otimes \omega(\pi)^{-1}$. On pose

$$
\Lambda^{\prime}(s, \pi)=N(\pi)^{s / 2} L(s, \pi) .
$$

Alors la fonction $\Lambda$ est une fonction holomorphe de $s$ et vérifie l'équation fonctionnelle:

$$
\Lambda(1-s, \pi)=W(\pi) \Lambda(s, \tilde{\pi}) .
$$

3.3. Le théorème fondamental. Par la théorie du corps de classes, une représentation linéaire continue de dimension 1 de $G_{Q}$ s'identifie avec un caractère de $A^{\times}$ trivial sur $\mathbb{Q}^{\times}$, donc aussi en composant avec le déterminant à une représentation de dimension 1 de $\mathrm{GL}\left(2, A_{\mathbb{Q}}\right)$. 
THÉORÈME. Soit $\rho$ une représentation comme en 1) vérifiant la condition (A), alors il existe $\pi$ comme en 2) tel que pour tout $\chi$ vérifiant (A):

$$
\Lambda(s, \rho \otimes \chi)=\Lambda(s, \pi \otimes \chi) .
$$

Ce résultat n'est qu'un cas particulier du célèbre théorème de Hecke-Weil-JacquetLanglands, pour lequel on réfère à la version améliorée obtenue par $\mathrm{W}$. $\mathrm{Li}([9])$.

Si $\pi$ et $\rho$ se correspondent comme ci-dessus, alors on a de plus:

$$
N(\pi)=N(\rho), \quad W(\pi)=W(\rho), \quad \omega(\pi)=\operatorname{dét}(\rho) .
$$

Il est connu que les classes d'isomorphie de $\pi$ et de $\rho$ sont déterminées par les fonctions $\Lambda(s, \rho \chi)$ et $\Lambda(s, \pi \chi)$ pour tout $\chi$ vérifiant (A). On ne sait pas si l'application $\rho \rightarrow \pi$, définie sur l'ensemble des représentations galoisiennes de dimension 2, de déterminant pair, vérifiant $(\mathrm{A})$, à valeurs dans l'ensemble des représentations automorphes paraboliques $\pi$ de $\mathrm{GL}\left(2, A_{\mathfrak{Q}}\right)$ telles que $\pi_{\infty}(C)=-1 / 4$ est une surjection.

Calcul de la constante d'Artin W( $(\rho)$, si $\rho$ est de conducteur $p$, et vérifie (A). Si $\rho$ est de conducteur $p$, on a vu que dét $\rho$ est aussi de conducteur $p$. Si $\rho$ vérife (A), soit $\pi$ l'image de $\rho$ par l'application ci-dessus. Pour tout nombre premier $q, \rho_{q}$ est de la série principale, obenue par le foncteur d'induction unitaire d'une représentation continue de dimension 1 , du groupe triangulaire supérieur:

$$
\left(\mu_{1} \otimes \mu_{2}\right)\left(\begin{array}{ll}
x & y \\
& z
\end{array}\right)=\mu_{1}(x) \mu_{2}(z)
$$

où $\mu_{1}, \mu_{2}$ sont deux caractères de $\mathbb{Q}_{a}^{\times}$; le couple non ordonné $\left(\mu_{1}, \mu_{2}\right)$ est déterminé par la représentation $\pi_{q}$. Si $q \neq p$, les deux caractères sont non ramifiés, tandis que si $q=p$, on peut supposer $\mu_{1}$ non ramifié, et

$$
\mu_{1} \mu_{2}=(\omega(\pi))_{p} .
$$

Il est connu que $W(\pi)$ est le produit de constantes locales

et que:

$$
W(\pi)=\prod W\left(\pi_{v}\right)
$$

$$
\begin{aligned}
& W\left(\pi_{\infty}\right)=(-1)^{a} \quad \text { ([7] p. 114) } \\
& W\left(\pi_{p}\right)=W\left(\mu_{1}\right) W\left(\mu_{2}\right),
\end{aligned}
$$

où $W(\mu)$ est une constante de module 1 , égale à 1 si $\mu$ est non ramifié, et

On en déduit:

$$
W(\mu) W\left(\mu^{-1}\right)=\mu(-1) .
$$

$$
(-1)^{a} W(\pi)=W\left(\omega(\pi)^{-1}\right)=W(\omega(\pi))^{-1} .
$$

Comme $W(\pi)=W(\rho)$ et $\omega(\pi)=$ dét $\rho$, on a:

LEMME. Si $\rho$ est de conducteur $p$, vérifie (A), et si $\rho(c)=(-1)^{a}$ id, alors

$$
W(\rho)=W(\text { dét } \rho)(-1)^{a} .
$$


En particulier, si dét $\rho$ est le symbole de Legendre, alors $W($ dét $\rho)=1$, (voir [10]) et

$$
W(\rho)=(-1)^{a} .
$$

REMARQUe. La théorie du nouveau vecteur ([5]) fait le lien entre les représentations automorphes de $\mathrm{GL}\left(2, A_{\mathbb{Q}}\right)$ et les formes modulaires classiques (holomorphes ou non). Elle permet de développer une théorie des formes primitives, pour les fromes modulaires non holomorphes, introduites par Maass, analogue à celle connue pour les formes classiques; dans ce langage, la forme primitive de Maass associée à $\rho$ vérifiant les conditions du théorème, avec

s'écrit:

$$
L(s, \rho)=\sum_{n \geqq 1} a(n) n^{-s} \quad \text { et } \quad \rho(c)=(-1)^{a}
$$

$$
f(x, y)=\sqrt{y} \sum_{\substack{n \in \mathbb{Z} \\ n \neq 0}} a(|n|)(\operatorname{sign} n)^{a} K_{0}(2 \pi|n| y) e^{2 i \pi n x}
$$

où $K_{0}$ est la fonction de Bessel définie par:

$$
K_{0}(x)=\int_{0}^{\infty}\left(t^{2}+1\right)^{-1 / 2} \cos (x t) d t, \text { si } x>0 .
$$

La fonction $f$ est propre pour le Laplacien hyperbolique

$$
\Delta=-y^{2}\left(d^{2} / d x^{2}+d^{2} / d y^{2}\right)
$$

de valeur propre $\lambda=1 / 4$.

Je remercie C. Bonnefont et $\mathrm{K}$. Ribet pour de nombreuses conversations intéressantes.

\section{BIBLIOGRAPHIE}

1. J. P. Buhler, Icosaedral Representations Springer-Verlag Lecture Notes 654 (1978).

2. Z. Borevich et I. Chafarevich, Théorie des nombres (Gauthiers-Villars, 1967).

3. A. Borel et H. Jacquet, Automorphic forms and automorphic representations (dans Automorphic Forms, Representations, and L-functions, AMS Proc. of Symp. in Pure Math. vol. XXXIII, part 1, p. 189-202 (1979)).

4. W. Casselman, $\mathrm{GL}_{n}$ dans Algebraic Number Fields, A. Fröhlich (Academic Press 1977).

5. W. Casselman, On some results of Atkin-Lehner, Math. Ann. 201 (1973), 301-314.

6. B. N. Delone et D. K. Faddev, The theory of irrationalities of the third degree Transl. of Math. Mon. vol. 10, AMS (1964).

7. S. Gelbart, Automorphic forms on adeles groups (Ann. of Math. Studies, $\mathrm{n}^{\circ} 23$, Princeton University Press 1975). 478-485.

8. H. J. Godwin, Real quartic fields with small discriminat, J. London Math. Soc. 31 (1956),

9. W. Li, On converse theorems for GL $\mathrm{GL}_{2}$ and $\mathrm{GL}_{1}$ Amer. J. of Math. 103 (1981), 851-885.

10. J. Martinet, Character Theory and Artin L-functions dans Algebraic Number Fields, A. Fröhlich (Academic Press 1977). 
11. J-P. Serre, Modular forms of weight one and Galois representations dans Algebraic Number Fields, A. Fröhlich (Academic Press 1977).

12. J. Tunnell, Artin's conjecture for representations of octaedral type Bull. Amer. Math. Soc. 5 (1981), 173-175.

ÉCOlE Normale SupÉrieure

92120 MONTROUGE

FRANCE 